\title{
Effect of Sarcopenia on Sleep Disturbance in Patients with Chronic Liver Diseases
}

\author{
Hiroki Nishikawa, Hirayuki Enomoto *, Kazunori Yoh, Yoshinori Iwata, Yoshiyuki Sakai, \\ Kyohei Kishino, Naoto Ikeda, Tomoyuki Takashima, Nobuhiro Aizawa, Ryo Takata, \\ Kunihiro Hasegawa, Noriko Ishii, Yukihisa Yuri, Takashi Nishimura, Hiroko Iijima \\ and Shuhei Nishiguchi
}
Division of Hepatobiliary and Pancreatic disease, Department of Internal Medicine, Hyogo College of Medicine, Nishinomiya, Hyogo 663-8501, Japan; nishikawa_6392@yahoo.co.jp (H.N.); mm2wintwin@ybb.ne.jp (K.Y.); yo-iwata@hyo-med.ac.jp (Y.I.); sakai429@hyo-med.ac.jp (Y.S.); hcm.kyohei@gmail.com (K.K.); nikeneko@hyo-med.ac.jp (N.I.); tomo0204@yahoo.co.jp (T.T.); nobu23hiro@yahoo.co.jp (N.A.); chano_chano_rt@yahoo.co.jp (R.T.); hiro.red1230@gmail.com (K.H.); ishinori1985@yahoo.co.jp (N.I.); gyma27ijo04td@gmail.com (Y.Y.); tk-nishimura@hyo-med.ac.jp (T.N.); hiroko-i@hyo-med.ac.jp (H.I.); nishiguc@hyo-med.ac.jp (S.N.)
* Correspondence: enomoto@hyo-med.ac.jp; Tel.: +81-798-45-6111; Fax: +81-798-45-6608

Received: 22 November 2018; Accepted: 20 December 2018; Published: 22 December 2018

\begin{abstract}
We sought to investigate the influence of sarcopenia as defined by muscle strength and skeletal muscle mass (SMM) on sleep disturbance as evaluated by the Japanese version of Pittsburgh Sleep Quality Index (PSQI-J) in chronic liver diseases (CLDs) $(n=419)$. Muscle strength and muscle mass were determined by grip strength (GS) and SMM using bioimpedance analysis. Patients were classified into four types: type A $(n=61)$; decreased GS and decreased SMM; type B $(n=45)$; decreased GS and non-decreased SMM; type C $(n=102)$; non-decreased GS and decreased SMM; and type $\mathrm{D}(n=211)$; non-decreased GS and non-decreased SMM. Factors associated with PSQI-J score 6 or more were examined. PSQI-J score 0-5 (normal) was found in $253(60.4 \%)$; 6-8 (mild) in $97(23.2 \%)$; 9-11 (moderate) in $45(10.7 \%)$ and 12 or more (severe) in 24 (5.7\%). Univariate analysis identified three factors to be significantly associated with PSQI-J score 6 or more: presence of liver cirrhosis (LC) $(P=0.0132)$; our classification of type A; B; C and D $(P<0.0001)$ and serum albumin level $(P=0.0041)$. Multivariate analysis showed that type $\mathrm{A}(P=0.0021)$ and type $\mathrm{B}(P=0.0220)$ were significant independent factors. In conclusion, sarcopenia in CLDs appears to be closely associated with sleep disturbance mainly due to muscle strength decline.
\end{abstract}

Keywords: chronic liver disease; sleep disturbance; pittsburgh sleep quality index; sarcopenia; grip strength

\section{Introduction}

Sleep is essential for mental and physical health and there is growing interest in sleep worldwide. Liver cirrhosis (LC) patients frequently describe sleep problems and non-LC patients with chronic liver diseases (CLDs) are not exceptions [1-11]. The mechanisms of sleep disturbance in CLD patients are not well elucidated; however, they appear temporally to be associated with CLD itself [12]. Persistent elevated melatonin levels due to impaired melatonin metabolism in LC patients can lead to disrupted circadian rhythms $[13,14]$. Sleep disturbances negatively impact innate immunity and are commonly associated with neurocognitive alterations in CLD patients regardless of the severity of liver fibrosis [2,15]. They include difficulty with falling asleep, fragmented sleep in night-time and increase in daytime somnolence, and have been suggested to adversely affect the quality of life (QOL) 
in CLD patients [8,15-17]. Thus, examining factors associated with sleep disturbances in CLD patients is clinically meaningful. Currently, one of extensively used and well validated patient-reported sleep questionnaires is Pittsburgh Sleep Quality Index (PSQI) [18-20].

On the other hand, sarcopenia is a disease entity combining poor muscle function and diminished skeletal muscle mass (SMM), which results in frailty, cachexia, and osteoporosis [21-32]. It can also be associated with worse patient QOL, prognosis and higher health care costs in various diseases including CLDs [22,24,26]. Increase of SMM is generally observed until 20 years and SMM reaches a peak between 20 and 50 years. Thereafter, it decreases by approximately $1 \%$ after the age of 50 years because of qualitative and quantitative changes in muscle fibers [33]. Sarcopenia is the main component of poor nutrition and is primarily responsible for the adverse clinical consequences seen in CLD patients $[22,24,26]$. In 2016, the Japanese Society of Hepatology (JSH) established the original criteria for liver diseases-related sarcopenia based on the Asian criteria for sarcopenia [21,25]. The JSH criteria adopts grip strength (GS) for the evaluation of muscle strength and bioimpedance analysis (BIA) and/or computed tomography for the evaluation of muscle mass, while unlike the Asian criteria for sarcopenia, there is no age restriction (i.e., 65 years old or more) in the JSH criteria for the evaluation of sarcopenia since CLD patients can have diminished SMM owing to impaired protein synthesis regardless of age [21].

However, as far as we are aware, data for the relevance between sarcopenia and sleep disturbances in CLD patients remain limited. In the current study, we sought to investigate the influence of sarcopenia on sleep disturbance as evaluated by the Japanese version of PSQI (PSQI-J) in patients with CLDs [20].

\section{Patients and Methods}

\subsection{Patients}

This study was a single-center retrospective study. A total of 440 CLD patients with data for GS, SMM using BIA and the PSQI available were admitted to our hospital between November 2013 and August 2018. Fifteen patients with underlying diseases such as overt hepatic encephalopathy, advanced malignancies, severe inflammatory diseases, or severe psychiatric diseases were excluded because they potentially affected the interpretation of PSQI score. As overestimates could occur for the calculation of skeletal muscle mass index (SMI) using BIA in patients with massive ascites, 6 subjects with severe ascites were also excluded from the study [22]. Four-hundred and 19 patients were thus analyzed. There were $156 \mathrm{LC}$ patients (37.2\%). In 71 LC patients, LC was determined by histological and imaging findings, and in the remaining 85 patients, $\mathrm{LC}$ was determined by imaging findings alone.

\subsection{Questionnaire Survey}

Sleep quality was assessed using PSQI-J as a screening tool for sleep disturbance [18-20]. The questionnaire consists of a total of 10 queries that form 7 categories: (i) subjective sleep quality, (ii) sleep latency, (iii) sleep duration, (iv) habitual sleep efficiency, (v) sleep disorders, (vi) use of sleep medications and (vii) daytime disturbance. Each category is rated on a scale of 0 to 3, and the sum of PSQI-J scores for all categories is 21 points. Higher PSQI-J scores suggest a poorer sleep quality. Favorable sensitivity and specificity were observed when the sum of PSQI-J scores exceeded 6 points [19]. Our study subjects were categorized as normal (PSQI-J score, 0-5), and the severity of sleep disturbance as mild (PSQI-J score, 6-8), moderate (PSQI-J score, 9-11) and severe (PSQI-J score, 12 or more) [18-20]. 


\subsection{Measurement of GS and SMI and our Study}

GS was measured according to the current guidelines [21]. Two measurements of GS were performed on both the left and right sides. The better measurement on each side is used, and GS was calculated as the mean of these values. SMI was defined as "appendicular SMM/(height $(\mathrm{m}))^{2}$ " using BIA. According to the current guidelines, patients with decreased GS (D-GS) were defined as those with GS $<26 \mathrm{~kg}$ for men and $<18 \mathrm{~kg}$ for women. Similarly, patients with decreased SMM (D-SMM) were defined as those with SMI $<7.0 \mathrm{~kg} / \mathrm{m}^{2}$ for men and $<5.7 \mathrm{~kg} / \mathrm{m}^{2}$ for women [21]. Again, the JSH criteria for sarcopenia in liver disease determines sarcopenia based on muscle strength and muscle mass irrespective of age considering the possibility that younger advanced LC patients suffer from sarcopenia [21]. In male, patients with GS $<26 \mathrm{~kg}$ and SMI $<7.0 \mathrm{~kg} / \mathrm{m}^{2}$ were classified as type A (sarcopenia), those with GS $<26 \mathrm{~kg}$ and SMI $\geq 7.0 \mathrm{~kg} / \mathrm{m}^{2}$ as type B, those with GS $\geq 26 \mathrm{~kg}$ and SMI $<7.0 \mathrm{~kg} / \mathrm{m}^{2}$ as type $\mathrm{C}$, and those with GS $\geq 26 \mathrm{~kg}$ and SMI $\geq 7.0 \mathrm{~kg} / \mathrm{m}^{2}$ as type D. In female, patients with GS $<18 \mathrm{~kg}$ and SMI $<5.7 \mathrm{~kg} / \mathrm{m}^{2}$ were classified as type A (sarcopenia), those with GS $<18 \mathrm{~kg}$ and SMI $\geq 5.7 \mathrm{~kg} / \mathrm{m}^{2}$ as type B, those with GS $\geq 18 \mathrm{~kg}$ and $\mathrm{SMI}<5.7 \mathrm{~kg} / \mathrm{m}^{2}$ as type $\mathrm{C}$, and those with GS $\geq 18 \mathrm{~kg}$ and $\mathrm{SMI} \geq 5.7 \mathrm{~kg} / \mathrm{m}^{2}$ as type D (Table 1 ).

Table 1. Our type classification stratified by grip strength (GS) and skeletal muscle mass index (SMI).

\begin{tabular}{ccccc}
\hline & \multicolumn{2}{c}{ Male } & \multicolumn{2}{c}{ Female } \\
\cline { 2 - 5 } & GS $<\mathbf{2 6} \mathbf{~ k g}$ & SMI $<\mathbf{7 . 0} \mathbf{~ k g / \mathbf { m } ^ { \mathbf { 2 } }}$ & $\mathbf{G S}<\mathbf{1 8} \mathbf{~ k g}$ & $\mathbf{S M I}<\mathbf{5 . 7} \mathbf{~ k g} / \mathbf{m}^{\mathbf{2}}$ \\
\hline Type A & Yes & Yes & Yes & Yes \\
Type B & Yes & No & Yes & No \\
Type C & No & Yes & No & Yes \\
Type D & No & No & No & No \\
\hline
\end{tabular}

First, the impacts of GS and SMM on sleep disturbance as assessed by PSQI-J were examined for all cases and several subgroups according to the LC status, gender, and age. PSQI-J scores among 4 types (type A, B, C and D) were also compared. Subsequently, factors associated with PSQI-J score 6 or more (mild, moderate, or severe sleep disturbance) were studied using univariate and multivariate analyses. We received the ethical approval from ethics committee of our hospital (approval no, 2296). The protocol in the study strictly observed all regulations of the Declaration of Helsinki.

\subsection{Statistical Considerations}

Comparisons between groups were performed for all cases and several subgroups according to the LC status, gender, and age. As for continuous parameters, Student's $t$ test, Mann-Whitney U test, analysis of variance or Kruskal-Wallis test were employed to assess group difference, as applicable. In categorical parameters, Fisher's exact tests or Pearson $\chi^{2}$ test was employed to assess group difference, as applicable. Factors with $P<0.1$ linked to PSQI-J score 6 or more in the univariate analysis were subjected to the multivariate logistic regression analysis to identify candidate parameters. Unless otherwise mentioned, data were indicated as median value (range). The threshold for statistical significance was set at $P<0.05$. The JMP 13.2 (SAS Institute Inc., Cary, NC, USA) was employed to carry out statistical analysis. 


\section{Results}

\subsection{Patient Baseline Characteristics}

Baseline characteristics in our study ( $n=419,200$ men and 219 women, median age $=64$ years) were presented in Table 2. The number of patients according to PSQI-J score was shown in Figure 1. The median (range) PSQI-J score was 5 (0-18). PSQI-J score 0-5 (normal) was found in $253(60.4 \%)$, 6-8 (mild) in 97 (23.2\%), 9-11 (moderate) in 45 (10.7\%) and 12 or more (severe) in 24 (5.7\%) [11]. Sleep disturbance with PSQI-J score 6 or more was thus observed in 166 patients $(39.6 \%)$. The median (range) GS and SMI in male patients were $34.3 \mathrm{~kg}(6.6-57.8 \mathrm{~kg})$ and $7.5 \mathrm{~kg} / \mathrm{m}^{2}\left(5.2-11.0 \mathrm{~kg} / \mathrm{m}^{2}\right)$ and those in female patients were $20.1 \mathrm{~kg}(5.9-36.1 \mathrm{~kg})$ and $5.9 \mathrm{~kg} / \mathrm{m}^{2}\left(3.9-8.1 \mathrm{~kg} / \mathrm{m}^{2}\right)$. Sarcopenia (i.e., type A) as defined by the JSH criteria was observed in 27 male patients $(13.5 \%)$ and 34 female patients $(15.5 \%)$ [21]. Among type A, B, C and D, overall differences were noted with statistical significance in age, gender, presence of LC, body mass index (BMI), serum albumin, prothrombin time, platelet count, total cholesterol, estimated glomerular filtration rate and PSQI-J score (Table 2). The median (range) PSQI-J score in LC patients (5 (0-18)) was significantly higher than that in non-LC patients $(4(0-15))(P=0.0046)$, suggesting the poorer sleep quality in LC patients compared with non-LC patients. The median (range) PSQI-J score in male patients $(4(0-16))$ was significantly lower than that in female patients $(5(0-18))(P=0.0420)$. The median (range) PSQI-J score in patients aged 65 years or more $(4.5(0-18))$ was similar to that in patients aged less than 65 years $(5(0-18))(P=0.5118)$.

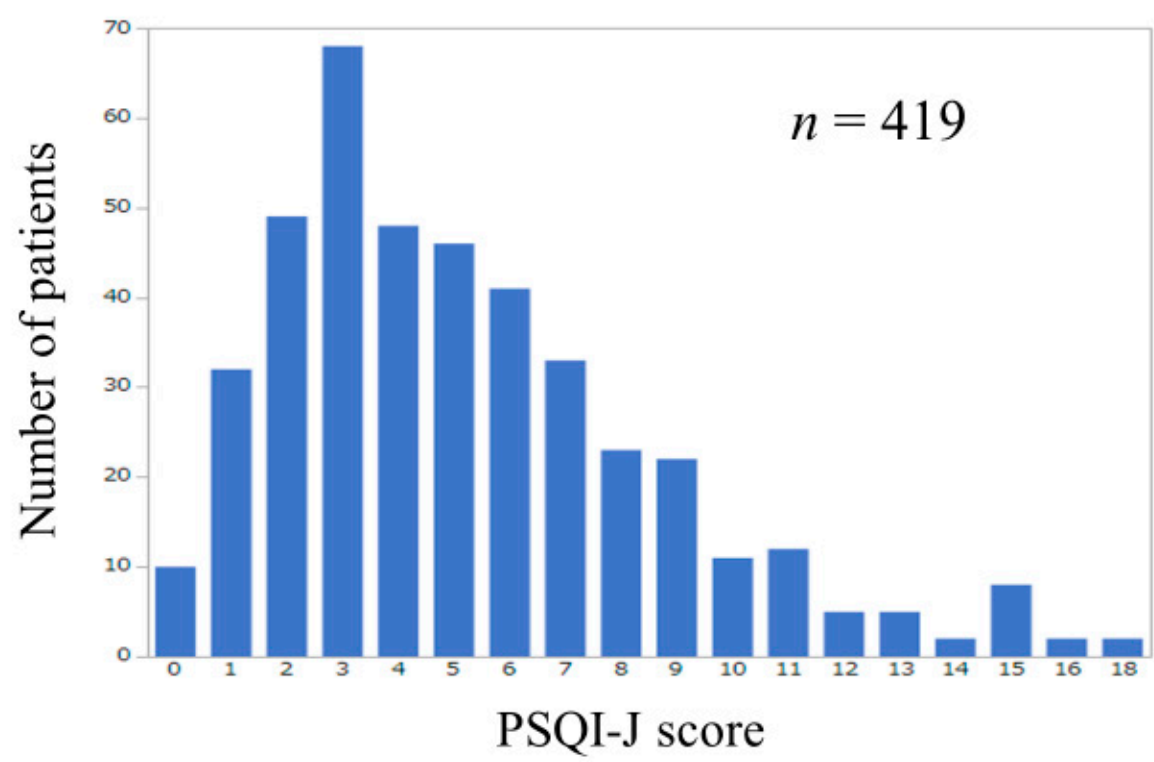

Figure 1. Number of patients according to PSQI-J score $(n=419)$. 
Table 2. Baseline characteristics.

\begin{tabular}{|c|c|c|c|c|c|c|}
\hline Variables & All Cases $(n=419)$ & Type A $(n=61)$ & Type B $(n=45)$ & Type C $(n=102)$ & Type $\mathrm{D}(n=211)$ & $\begin{array}{l}\text { Overall (Type A, B, C } \\
\text { and D) } P \text { Value }\end{array}$ \\
\hline Age (years) & $64(25-90)$ & $71(25-90)$ & $69(33-83)$ & $67(29-84)$ & $58(26-90)$ & $<0.0001$ \\
\hline Gender, male/female & $200 / 219$ & $27 / 34$ & $12 / 33$ & $62 / 40$ & $99 / 112$ & 0.0016 \\
\hline $\begin{array}{c}\mathrm{HBV} / \mathrm{HCV} / \mathrm{HBV} \text { and } \\
\mathrm{HCV} / \mathrm{NBNC}\end{array}$ & $61 / 259 / 8 / 91$ & $5 / 42 / 2 / 12$ & $7 / 28 / 0 / 10$ & $15 / 67 / 3 / 17$ & $34 / 122 / 3 / 52$ & 0.5540 \\
\hline Presence of LC, yes/no & $156 / 263$ & $30 / 31$ & $27 / 18$ & $31 / 71$ & $68 / 143$ & 0.0004 \\
\hline Body mass index $\left(\mathrm{kg} / \mathrm{m}^{2}\right)$ & $22.6(14.8-41.4)$ & $21.3(17.2-26.9)$ & $25.5(19.8-32.8)$ & $20.6(16.0-28.3)$ & $24.1(14.8-41.4)$ & $<0.0001$ \\
\hline Total bilirubin (mg/dL) & $0.8(0.3-5.6)$ & $0.7(0.4-2.7)$ & $0.85(0.3-4.0)$ & $0.9(0.3-2.4)$ & $0.8(0.3-5.6)$ & 0.1415 \\
\hline Serum albumin $(\mathrm{g} / \mathrm{dL})$ & $4.2(1.8-5.2)$ & $4.1(2.4-4.7)$ & $4.1(2.5-5.2)$ & $4.3(2.3-5.0)$ & $4.2(1.8-4.9)$ & $<0.0001$ \\
\hline Prothrombin time (\%) & $88.7(23.0-122.9)$ & $85.3(46.5-122.5)$ & $80.4(44.6-110.1)$ & $90.0(23.0-120.2)$ & $89.8(28.3-122.9)$ & 0.0002 \\
\hline $\begin{array}{l}\text { Platelet count } \\
\left(\times 10^{4} / \mathrm{mm}^{3}\right)\end{array}$ & $16.8(1.4-42.2)$ & $15.3(2.8-39.7)$ & $13.1(3.6-30.0)$ & $15.3(4.1-33.0)$ & $17.8(1.4-42.2)$ & 0.0012 \\
\hline Total cholesterol (mg/dL) & $177.5(80-420)$ & $175(80-420)$ & $158(88-244)$ & $182(99-290)$ & $181(90-292)$ & 0.0080 \\
\hline AST (IU/L) & $28(10-222)$ & $29(10-191)$ & $34(15-133)$ & $27(11-143)$ & $27(12-222)$ & 0.2732 \\
\hline ALT (IU/L) & $24(5-297)$ & $27(5-263)$ & $28(5-213)$ & $23(5-188)$ & $24(8-297)$ & 0.8079 \\
\hline $\operatorname{ALP}(\mathrm{IU} / \mathrm{L})$ & 239 (91-5065) & $253.5(121-5065)$ & $249(95-579)$ & $231(112-650)$ & $234(91-1206)$ & 0.1702 \\
\hline GGT (IU/L) & $28(7-542)$ & $26(8-462)$ & $37(11-311)$ & $26.5(10-386)$ & $27(7-542)$ & 0.8156 \\
\hline eGFR $\left(\mathrm{mL} / \mathrm{min} / 1.73 \mathrm{~m}^{2}\right)$ & $83(5-162)$ & $80(87-141)$ & $83.5(31-146)$ & $83(5-136)$ & $83.5(34-162)$ & 0.0497 \\
\hline HbA1c (NGSP) & $5.7(3.7-10.4)$ & $5.6(4.7-8.8)$ & $5.6(4.1-8.2)$ & $5.7(3.8-10.1)$ & $5.6(3.7-10.4)$ & 0.9597 \\
\hline Serum sodium (mmol/L) & $140(124-148)$ & $140(124-144)$ & $140(130-146)$ & $140(131-148)$ & $140(126-144)$ & 0.2000 \\
\hline PSQI-J score & $5(0-18)$ & $6(0-18)$ & $6(0-15)$ & $4(0-16)$ & $4(0-18)$ & 0.0006 \\
\hline
\end{tabular}

Data are expressed as median value (range). HBV; hepatitis B virus, HCV; hepatitis C virus, NBNC; non-B and non-C, LC; liver cirrhosis, AST; aspartate aminotransferase, ALT; alanine aminotransferase, ALP; alkaline phosphatase, GGT; gamma-glutamyltransferase, eGFR; estimated glomerular filtration rate, NSGP; National Glycohemoglobin Standardization Program, PSQI-J; Japanese version of Pittsburgh Sleep Quality Index. 


\subsection{Influence of GS and SMM on PSQI-J for All Cases $(n=419)$}

The median (range) PSQI-J score in the D-GS $(6(0-18), n=106)$ was significantly higher than that in the non-decreased GS (ND-GS) $(4(0-18), n=313)(P=0.0002)$, (Figure 2A). The median (range) PSQI-J score in the D-SMM (5 (0-18), $n=163)$ was similar to that in the non-decreased SMM (ND-SMM) (5 $(0-18), n=256)(P=0.3005)$, (Figure 2B). The median (range) PSQI-J score in type A (sarcopenia) (6 (0-18), $n=61$ ) was significantly higher than that in non-type A (i.e., type B, C and D) (5 (0-18), $n=358)(P=0.0031)$, (Figure 2C). In comparison among four types, the overall difference was observed with significance $(P=0.0006)$, (Figure $2 \mathrm{D})$.
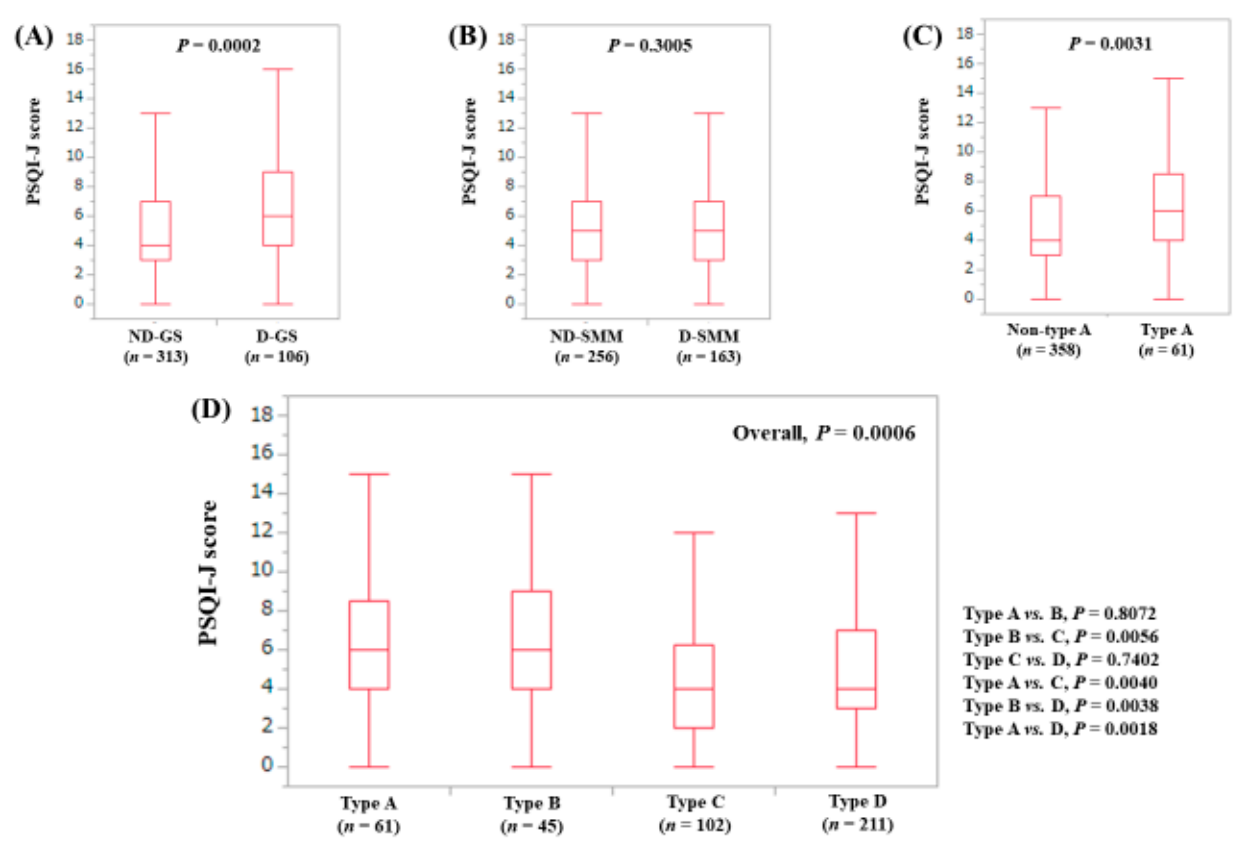

Figure 2. PSQI-J score stratified by GS and SMM for all cases $(n=419)$. (A) Non-decreased GS vs. decreased GS. (B) Non-decreased SMM vs. decreased SMM. (C) Non-type A (type B, C and D) vs. type A. (D) Comparison among four types (type A, B, C and D).

\subsection{Stratified Analysis 1: Influence of GS and SMM on PSQI-J for LC Patients ( $n=156$ )}

The median (range) PSQI-J score in the D-GS (6 (0-18), $n=57)$ was significantly higher than that in the ND-GS $(4(0-18), n=99)(P=0.0102)$, (Figure 3A). The median (range) PSQI-J score in the D-SMM (5 (0-18), $n=61)$ was similar to that in the ND-SMM (5 $(0-18), n=95)(P=0.6108)$, (Figure 3B). The median (range) PSQI-J score in type A $(7(0-18), n=30)$ tended to be significantly higher compared with non-type A (5 $(0-18), n=126)(P=0.0793)$, (Figure $3 C)$. In comparison among four types, the overall difference was noted with significance $(P=0.0286)$, (Figure 3D). 

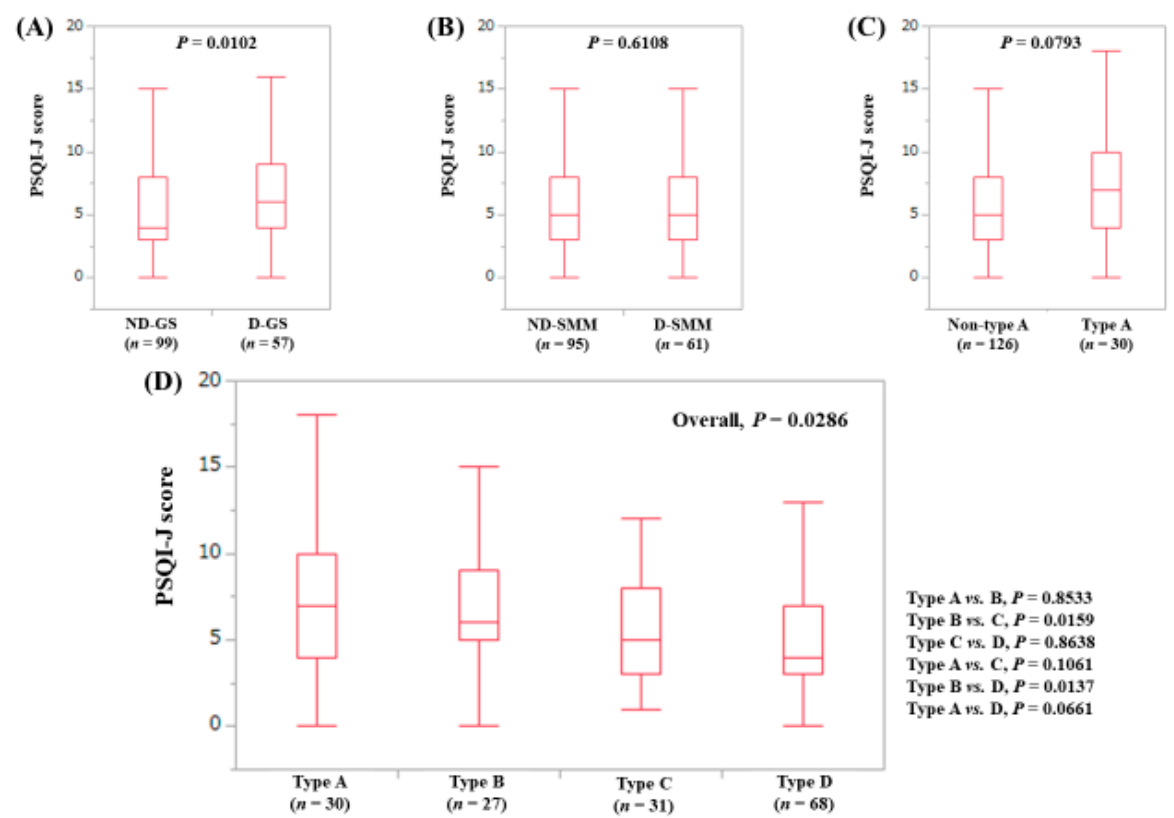

Figure 3. PSQI-J score stratified by GS and SMM for LC cases $(n=156)$. (A) Non-decreased GS vs. decreased GS. (B) Non-decreased SMM vs. decreased SMM. (C) Non-type A (type B, C and D) vs. type A. (D) Comparison among four types (type A, B, C and D).

\subsection{Stratified Analysis 2: Influence of GS and SMM on PSQI-J for Non-LC Patients ( $n=263$ )}

The median (range) PSQI-J score in the D-GS $(6(0-13), n=49)$ had a tendency for significance compared with the ND-GS $(4(0-15), n=214)(P=0.0667)$, (Figure 4A). The median (range) PSQI-J score in the D-SMM (4 (0-15), $n=102)$ was equal to that in the ND-SMM $(4(0-15), n=161)(P=0.3493)$, (Figure 4B). The median (range) PSQI-J score in type A (6 (0-13), $n=31)$ was significantly higher compared with non-type A $(4(0-15), n=232)(P=0.0454)$, (Figure $4 C)$. In comparison among four types, the overall difference was noted with a trend for significance $(P=0.0841)$, (Figure $4 \mathrm{D}$ ).
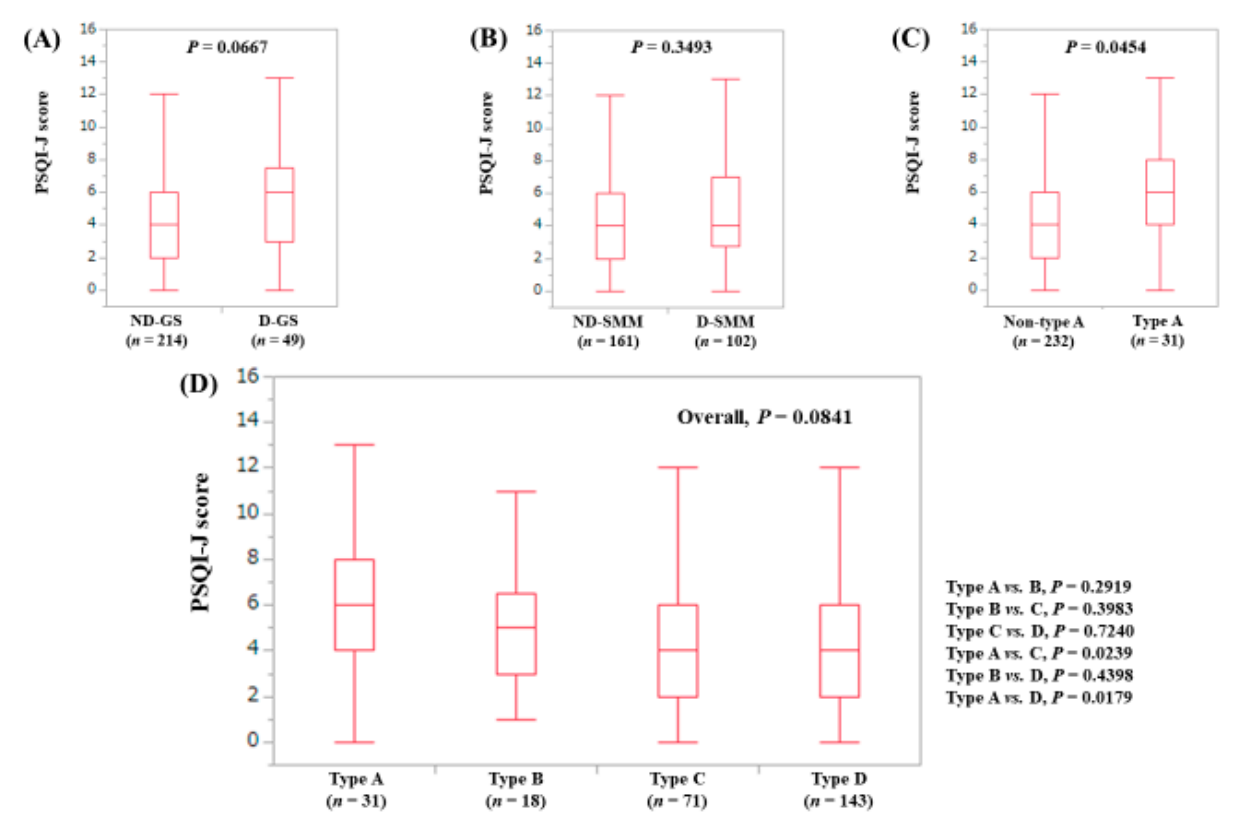

Type A rr. B, $P-0.2919$
Type B vs, C, $P-0.3983$

Type B vs. C, $P-0.3983$

Type C rs. D, $P=0.7240$

Type A rs, C, $P=0.0239$

Type B vs. D, $P=0.4398$
Type A rs. D, $P=0.0179$

Figure 4. PSQI-J score stratified by GS and SMM for non-LC cases $(n=263)$. (A) Non-decreased GS vs. decreased GS. (B) Non-decreased SMM vs. decreased SMM. (C) Non-type A (type B, C and D) vs. type A. (D) Comparison among four types (type A, B, C and D). 


\subsection{Stratified Analysis 3: Influence of GS and SMM on PSQI-J for Male Patients $(n=200)$}

The median (range) PSQI-J score in the D-GS $(6(0-15), n=39)$ was significantly higher compared with the ND-GS $(4(0-16), n=161)(P=0.0051)$, (Figure 5A). The median (range) PSQI-J score in the D-SMM (4 (0-16), $n=89)$ was identical to that in the ND-SMM $(4(0-15), n=111)(P=0.2502)$, (Figure $5 \mathrm{~B})$. The median (range) PSQI-J score in type A $(7(0-15), n=27)$ was significantly higher than that in non-type A $(4(0-16), n=173)(P=0.0101)$, (Figure 5C). In comparison among four types, the overall difference was identified with significance $(P=0.0196)$, (Figure $5 \mathrm{D})$.
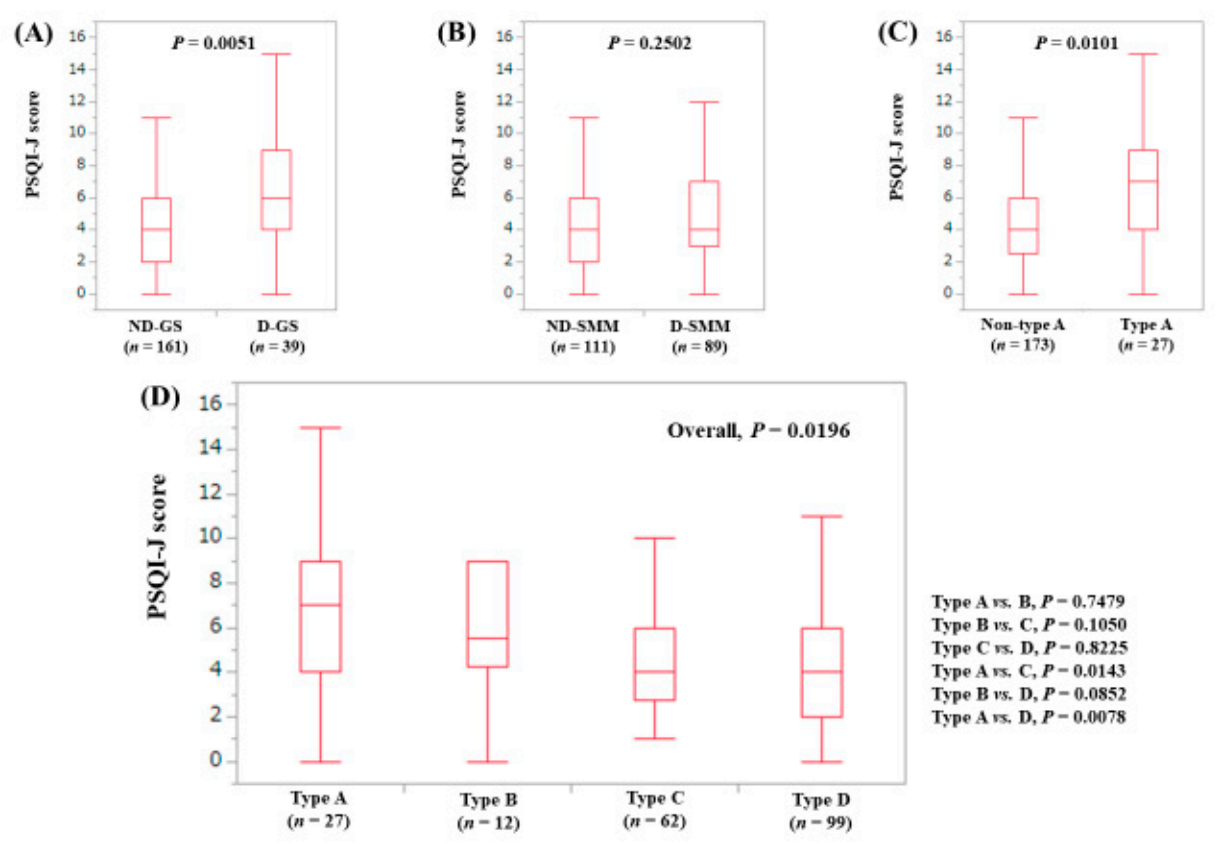

Figure 5. PSQI-J score stratified by GS and SMM for male cases $(n=200)$. (A) Non-decreased GS vs. decreased GS. (B) Non-decreased SMM vs. decreased SMM. (C) Non-type A (type B, C and D) vs. type A. (D) Comparison among four types (type A, B, C and D).

\subsection{Stratified Analysis 4: Influence of GS and SMM on PSQI-J for Female Patients ( $n=219)$}

The median (range) PSQI-J score in the D-GS $(6(0-18), n=67)$ was significantly higher compared with the ND-GS $(5(0-18), n=152)(P=0.0256)$, (Figure 6A). The median (range) PSQI-J score in the D-SMM (5 (0-18), $n=74)$ was similar to that in the ND-SMM $(5(0-18), n=145)(P=0.4966)$, (Figure 6B). The median (range) PSQI-J score in type A $(6(0-18), n=34)$ had a trend for significance compared to non-type A $(5(0-18), n=185)(P=0.0974)$, (Figure 6C). In comparison among four types, the overall difference was noted with a tendency for significance $(P=0.0835)$, (Figure 6D). 

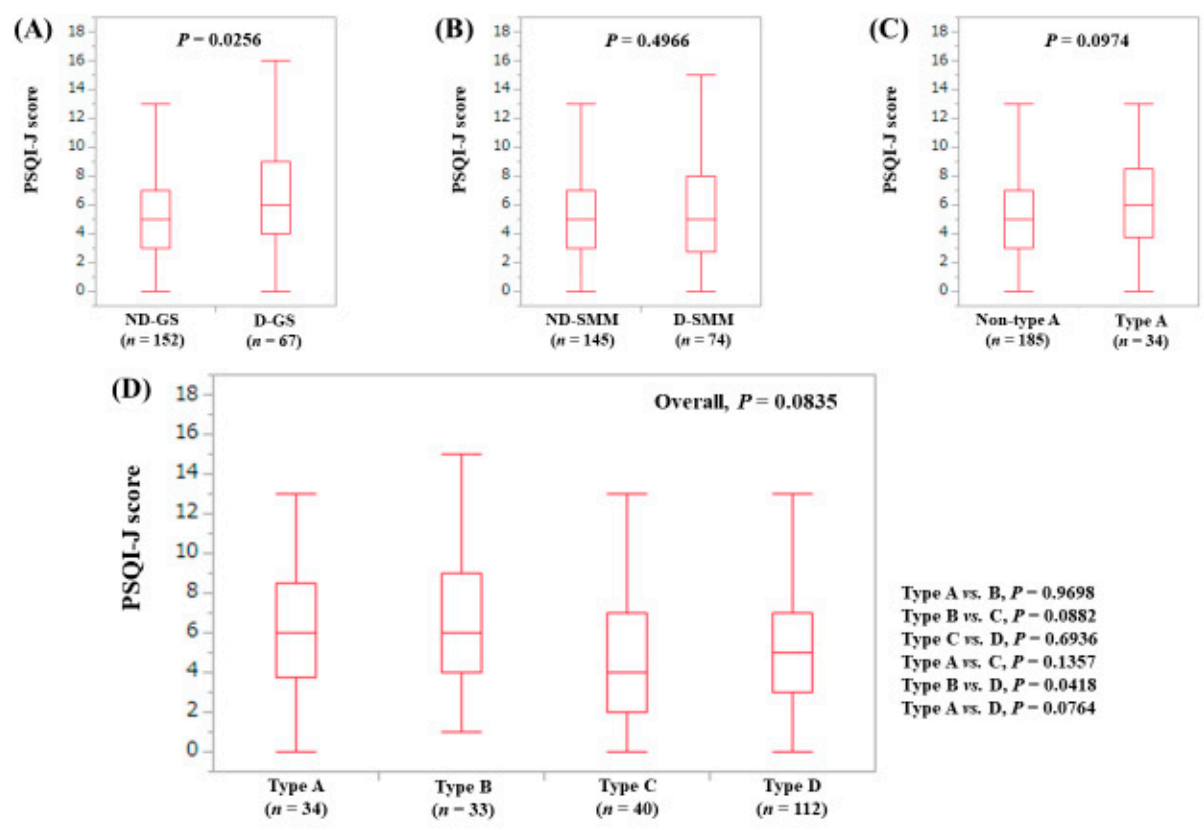

Figure 6. PSQI-J score stratified by GS and SMM for female cases $(n=219)$. (A) Non-decreased GS vs. decreased GS. (B) Non-decreased SMM vs. decreased SMM. (C) Non-type A (type B, C and D) vs. type A. (D) Comparison among four types (type A, B, C and D).

\subsection{Stratified Analysis 5: Influence of GS and SMM on PSQI-J for Patients Aged 65 Years or More $(n=202)$}

The median (range) PSQI-J score in the D-GS $(6(0-18), n=75)$ tended to be significantly higher than that in the ND-GS $(4(0-16), n=127)(P=0.0665)$, (Figure 7A). The median (range) PSQI-J score in D-SMM (5 (0-18), $n=108)$ was equal to that in the ND-SMM (4 (0-15), $n=94)(P=0.2580)$, (Figure 7B). The median (range) PSQI-J score in type A $(6(0-18), n=46)$ was significantly higher compared with non-type A (4 (0-16), $n=156)(P=0.0380)$, (Figure 7C). In comparison among four types, the overall difference was not significant $(P=0.1439)$, (Figure 7D).
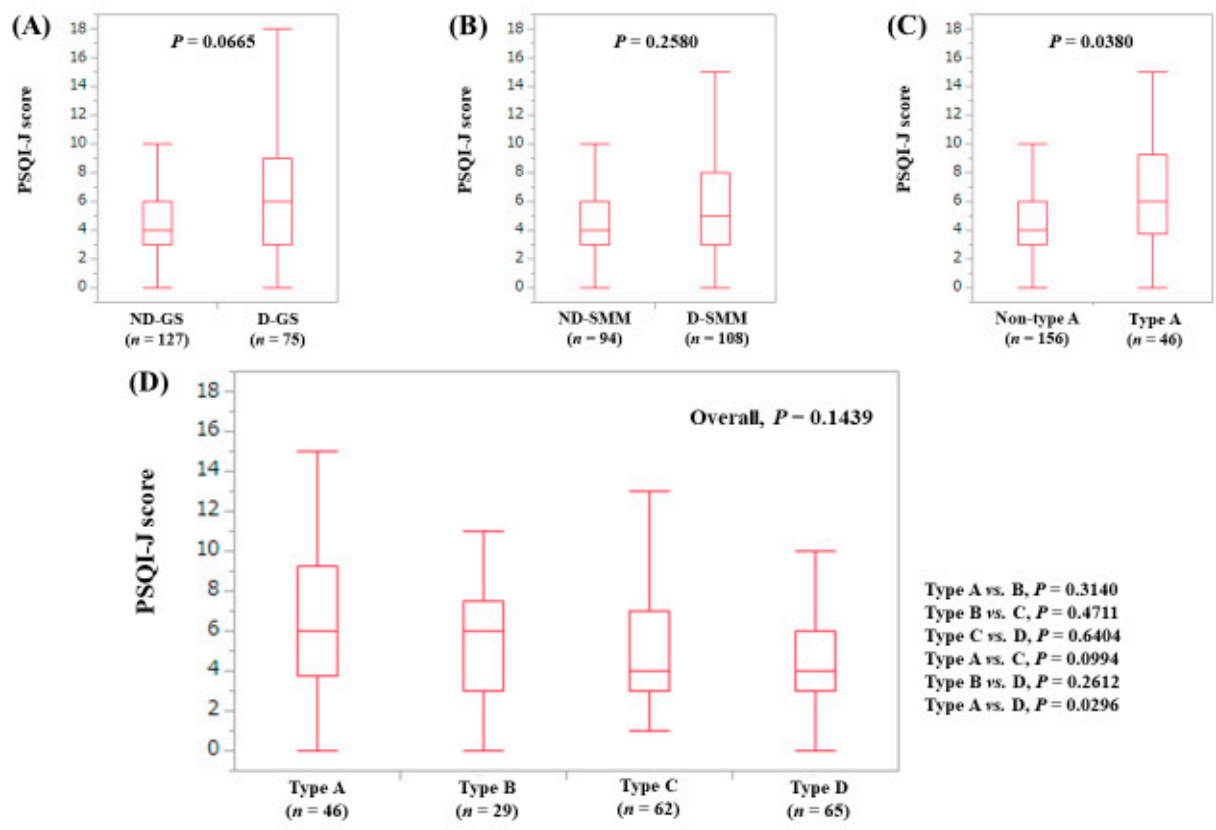

Type A rs. B, $P=0.3140$
Type B vs. C, $P=0.4711$

Type C rs. D, $P=0.6404$

Type A rs. C, $P=0.0994$

Type B vs. D, $P=0.2612$

Type A n. D, $P=0.0296$

Figure 7. PSQI-J score stratified by GS and SMM for patients aged 65 years or more $(n=202)$. (A) Non-decreased GS vs. decreased GS. (B) Non-decreased SMM vs. decreased SMM. (C) Non-type A (type B, C and D) vs. type A. (D) Comparison among four types (type A, B, C and D). 
3.8. Stratified Analysis 6: Influence of GS and SMM on PSQI-J for Patients Aged Less Than 65 Years $(n=217)$

The median (range) PSQI-J score in the D-GS $(7(2-16), n=31)$ was significantly higher than that in the ND-GS $(4(0-18), n=186)(P=0.0002)$. (Figure 8A) The median (range) PSQI-J score in the D-SMM $(4(0-16), n=55)$ was identical to that in the ND-SMM $(5(0-18), n=162)(P=0.6931)$. (Figure 8B) The median (range) PSQI-J score in type A $(7(2-16), n=15)$ was significantly higher compared with non-type A (4 (0-18), $n=202)(P=0.0295)$. (Figure $8 C)$ In comparison among four types, the overall difference was noted with significance $(P=0.0002)$. (Figure $8 \mathrm{D})$.
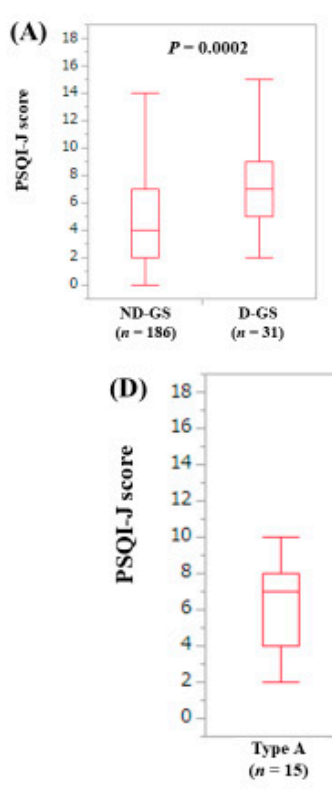

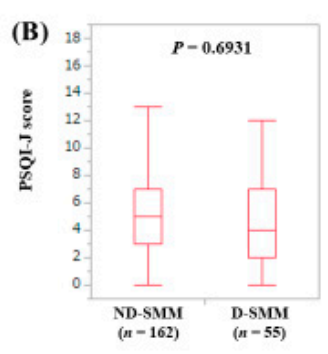

Overall, $P=0.0002$

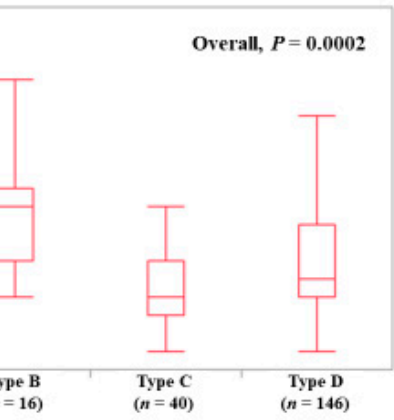

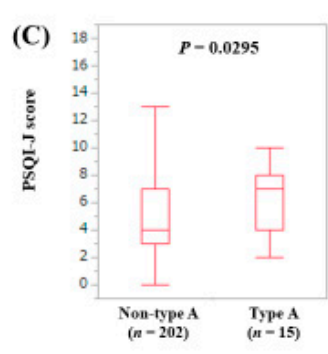

Type A vs. B, $P=0.3228$

Type B rs. C, $P=0.0004$

Type A vs. C, $P=0.009$

Type B rs. D, $P=0.0005$
Type A vs. D, $P=0.0203$

Figure 8. PSQI-J score stratified by GS and SMM for patients less than 65 years $(n=217)$. (A) Non-decreased GS vs. decreased GS. (B) Non-decreased SMM vs. decreased SMM. (C) Non-type A (type B, C and D) vs. type A. (D) Comparison among four types (type A, B, C and D).

\subsection{Univariate and Multivariate Analyses of Factors Associated with PSQI-J Score 6 or More}

Univariate analysis identified three factors to be significantly associated with PSQI-J score 6 or more: presence of LC $(P=0.0132)$, our classification of type A, B, C and D $(P<0.0001)$ and serum albumin level $(P=0.0041)$, while gender tended to be significant $(P=0.0721)$, (Table 3$)$. Multivariate analysis for the four factors showed that type $\mathrm{A}(P=0.0021)$ and type $\mathrm{B}(P=0.0220)$ were significant factors linked to PSQI-J score 6 or more (Table 4). Hazard ratios (HRs) and 95\% confidence intervals for these factors are listed in Table 4.

Table 3. Univariate analyses of factors linked to PSQI-J score 6 or more.

\begin{tabular}{cccc}
\hline Variables & $\begin{array}{c}\text { PSQI-J Score 6 or More } \\
(\boldsymbol{n}=\mathbf{1 6 6})\end{array}$ & $\begin{array}{c}\text { PSQI-J Score Less than 6 } \\
(\boldsymbol{n}=\mathbf{2 5 3})\end{array}$ & $\boldsymbol{P}$ Value \\
\hline Age (years) & $65(26-90)$ & $62(25-90)$ & 0.2326 \\
Gender, male/female & $70 / 96$ & $130 / 123$ & 0.0721 \\
HBV/HCV/HBV and & $21 / 106 / 3 / 36$ & $40 / 153 / 5 / 55$ & 0.8294 \\
HCV/NBNC & $22.9(16.0-41.4)$ & $22.5(14.8-36.5)$ & 0.1589 \\
Body mass index (kg/m $\left.{ }^{2}\right)$ & $74 / 92$ & $82 / 171$ & 0.0132 \\
Presence of LC, yes/no & $36 / 26 / 32 / 72$ & $25 / 19 / 70 / 139$ & $<0.0001$ \\
Type, A/B/C/D & $0.8(0.3-5.6)$ & $0.8(0.3-4.0)$ & 0.6316 \\
Total bilirubin (mg/dL) & $4.1(1.8-5.0)$ & $4.2(2.3-5.2)$ & 0.0041 \\
Serum albumin (g/dL) & $87.5(38.4-117.6)$ & $90.0(23.0-122.9)$ & 0.1593 \\
Prothrombin time (\%) & &
\end{tabular}


Table 3. Cont.

\begin{tabular}{cccc}
\hline Variables & $\begin{array}{c}\text { PSQI-J Score } \mathbf{6} \text { or More } \\
(\boldsymbol{n}=\mathbf{1 6 6})\end{array}$ & $\begin{array}{c}\text { PSQI-J Score Less than } \mathbf{6} \\
(\boldsymbol{n}=\mathbf{2 5 3})\end{array}$ & $\boldsymbol{P}$ Value \\
\hline Platelet count $\left(\times 10^{4} / \mathrm{mm}^{3}\right)$ & $16.5(2.8-42.2)$ & $16.9(1.4-37.6)$ & 0.5222 \\
AST (IU/L) & $29.5(12-191)$ & $28(10-222)$ & 0.6180 \\
ALT (IU/L) & $25(5-297)$ & $24(5-213)$ & 0.5807 \\
ALP (IU/L) & $235(105-5065)$ & $244(91-1206)$ & 0.9472 \\
GGT (IU/L) & $28.5(8-462)$ & $27(7-542)$ & 0.5677 \\
Total cholesterol (mg/dL) & $175.5(80-420)$ & $179.5(88-290)$ & 0.3166 \\
eGFR (mL/min/1.73m $\left.{ }^{2}\right)$ & $83(83-162)$ & $83(5-147)$ & 0.9792 \\
Serum sodium $(\mathrm{mmol} / \mathrm{L})$ & $140(124-148)$ & $140(131-146)$ & 0.6521 \\
HbA1c $(\mathrm{NGSP})$ & $5.7(3.7-10.1)$ & $5.7(3.8-10.4)$ & 0.7734 \\
\hline
\end{tabular}

PSQI-J: Japanese version of Pittsburgh Sleep Quality Index, HBV: hepatitis B virus, HCV: hepatitis C virus, NBNC: non-B and non-C, LC: liver cirrhosis, AST: aspartate aminotransferase, ALT: alanine aminotransferase, ALP: alkaline phosphatase, GGT: gamma-glutamyltransferase, eGFR: estimated glomerular filtration rate, NSGP: National Glycohemoglobin Standardization Program PSQI-J: Japanese version of Pittsburgh Sleep Quality Index

Table 4. Multivariate analyses of factors linked to PSQI-J score 6 or more.

\begin{tabular}{cccc}
\hline & \multicolumn{3}{c}{ Multivariate Analysis } \\
\hline & Hazard Ratio & $\mathbf{9 5 \%}$ CI & P Value \\
\hline Presence of LC & & \\
\hline Yes & 0.796 & $0.483-1.310$ & 0.3704 \\
No & 1.000 & Reference & \\
\hline Gender & & $0.900-2.082$ & 0.1412 \\
Male & 1.369 & Reference & \\
Female & 1.000 & $0.831-2.095$ & 0.2390 \\
\hline Serum albumin (per 1.0 g/dL) & 1.320 & & \\
\hline Type & & $0.217-0.713$ & 0.0021 \\
A (D-GS and D-SMM) & 0.393 & $0.230-0.892$ & 0.0220 \\
B (D-GS and ND-SMM) & 0.453 & $0.609-1.710$ & 0.9386 \\
D (ND-GS and D-SMM) & 1.020 & Reference & \\
\hline
\end{tabular}

PSQI-J: Japanese version of Pittsburgh Sleep Quality Index, LC: liver cirrhosis, D-GS: decreased grip strength, D-SMM: decreased skeletal muscle mass, ND-GS: non-decreased grip strength, ND-SMM: non-decreased skeletal muscle mass, CI: confidence interval

\section{Discussion}

Sleep disruption entails a heavy burden, and adversely impacts patient QOL, mood alterations, and possibly fatigue, which can be related to unfavorable clinical outcomes $[10,11,15]$. To our knowledge, this is the first study demonstrating the relevance between sleep disturbance and sarcopenia in CLD patients. To clarify these clinical questions is of importance because liver disease-related sarcopenia has been gaining much caution these days due to its significant correlation with QOL and prognosis [21-31].

In our results, compared with ND-GS patients, D-GS patients had higher PSQI-J scores with statistical significance or near significance for all analyses, while PSQI-J scores in patients with D-SMM were identical to those with ND-SMM for all analyses. Additionally, type A (sarcopenia) and type B (D-GS and ND-SMM) were independent predictors linked to PSQI-J score 6 or more in the multivariate analysis ( $\mathrm{HR}=0.393$ in the type $\mathrm{A}$ and $\mathrm{HR}=0.453$ in the type $\mathrm{B}$ (type $\mathrm{D}$ as a reference)). These results denoted that sarcopenia in CLDs was closely associated with sleep disturbance and this was mainly influenced by muscle strength decline and SMM itself did not affect sleep quality. In this respect, our study results appear to shed some insights on the better understanding of sarcopenia and sleep disturbance in CLD patients. For sarcopenic CLD patients with sleep disturbance, physical exercise may 
be useful [34]. Recent reports trend towards a beneficial effect of physical exercise with improvement in QOL [34].

CLD patients are aging these days in our country and aging itself can cause sleep disturbance in older adults [35-38]. Serum melatonin levels, which regulate circadian rhythms such as the sleep-wake rhythm, vary considerably with aging [14]. Our data of median ages in the types of A, B, C, and D were $71,69,67$ and 58 years, respectively, indicating the impact of aging on sarcopenia. On the other hand, the PSQI-J score in patients aged 65 years or more was similar to that in patients aged less than 65 years $(P=0.5118)$ in this study. Type A and B patients tended to be older than other types and type A and B were independent predictors associated with PSQI-J score 6 or more. While type A and $\mathrm{B}$ patients had poorer liver function as reflected by lower serum albumin levels. In addition, in our univariate analysis, presence of LC and serum albumin were significant factors linked to PSQI-J score 6 or more, whereas age was not. In view of these results, impaired protein synthesis due to poor liver function rather than aging itself appears to cause sleep disturbances in CLD patients.

In our data, the median (range) PSQI-J score in LC patients (5 (0-18)) was significantly higher vs. non-LC patients $(4(0-15), P=0.0046)$ and the proportion of PSQI-J score 6 or more in LC and non-LC patients were $47.4 \%(74 / 156)$ and $35.0 \%(92 / 263)(P=0.0132)$. A recent study reported that $81 \%$ in LC patients had disturbed sleep with PSQI score 6 or more and sleep disruptions were associated with muscle cramps, increase in daytime somnolence, and poorer QOL [11]. Although the reasons for this discrepancy (i.e., $47.4 \%$ in our LC patients vs. $81 \%$ [11] in the frequency of disturbed sleep) are unclear, clinicians should be fully aware that sleep disturbance represents a pivotal unmet medical need in CLD patients [11]. On the other hand, the median (range) PSQI-J score in male (4 (0-16)) was significantly lower vs. female $(5(0-18))(P=0.0420)$, indicating poorer sleep quality in female patients. While gender was not an independent factor associated with PSQI-J 6 or more, gender difference for sleep disturbance cannot be ignored to provide CLD patients with individualized disease management [39].

In our subgroup analysis in patients aged less than 65 years $(n=217)$, overall difference in comparison among four types (type A, B, C and D) was noted with the strongest $P$ value $(P=0.0002)$. Of these 217 patients, 78 patients (35.9\%) and 15 patients (6.9\%) had PSQI-J score 6 or more and sarcopenia. Primary sarcopenia is an aging-related sarcopenia and secondary sarcopenia is a disease-specific sarcopenia [21]. As mentioned above, the current JSH criteria for sarcopenia in liver disease determines sarcopenia based on muscle strength and muscle mass irrespective of age. Our study data may support the validity of the current JSH guidelines for sarcopenia.

Obesity, insulin resistance and poor nutrition are reported to be associated with sleep disruptions $[9,40,41]$. Bernsmeier C, et al demonstrated that non-alcoholic fatty liver disease patients had shortened sleep duration, prolonged time to fall asleep, and poor sleep quality [9]. In our univariate analyses, serum albumin was significant linked to JSQI-J score 6 or more, while total cholesterol level, $\mathrm{BMI}$ and $\mathrm{HbA1c}$ were not. Additionally, in the subgroup analyses according to liver disease etiologies (hepatis B virus, hepatitis C virus and non-B and non-C), total cholesterol level, BMI and HbA1C were not significant linked to PSQI-J score 6 or more (data not shown). The reasons for these remain uncertain and further investigations will be necessary to confirm these results.

Our study was associated with several limitations. Firstly, the study was a single-center observational study with a retrospective nature. Secondly, PSQI is a subjective assessment method for sleep disturbance and objective assessment for sleep such as actigraphy was not performed in this study [4]. Thirdly, GS can vary depending on patient daily life activities. Fourthly, patients with massive ascites or overt hepatic encephalopathy who are potentially involved in liver disease-related sarcopenia were excluded due to the lack of reliability in BIA or patient-administered questionnaire, leading to bias. Fifthly, data for alcohol use are lacking and patients with minimal hepatic encephalopathy which potentially affects PSQI-J score may be included in our study subjects, also creating bias [42]. Finally, it was unclear as to whether sarcopenia caused sleep disturbance or whether sleep disturbance caused sarcopenia in this study. Caution should be therefore exercised for 
the interpretation of our data. Nevertheless, our study results indicated that sarcopenia in CLDs was closely associated with sleep disturbance mainly due to muscle strength decline.

\section{Conclusions}

Sarcopenia can be an independent predictor for sleep disturbance in patients with CLDs. Clinicians should be aware of the presence of sarcopenia in CLD patients with sleep disturbance.

Author Contributions: Data curation, K.Y., Y.I., Y.S., K.K., N.I. (Naoto Ikeda), T.T., N.A., R.T., K.H., N.I. (Noriko Ishii), Y.Y., T.N. and H.I.; Supervision, S.N.; Writing—original draft, H.N.; Writing—review \& editing, H.E.

Funding: This research received no external funding.

Acknowledgments: The authors gratefully thank all medical staff in our nutritional guidance room for their help with data collection.

Conflicts of Interest: None of the authors has any conflicts of interest to declare.

\section{References}

1. Anderson, K.; Jones, D.E.; Wilton, K.; Newton, J.L. Restless leg syndrome is a treatable cause of sleep disturbance and fatigue in primary biliary cirrhosis. Liver Int. 2013, 33, 239-243. [CrossRef] [PubMed]

2. Ozsahin, M.; Gonen, I.; Ermis, F.; Oktay, M.; Besir, F.H.; Kutlucan, A.; Sahin, A.; Ataoglu, S. The prevalence of fibromyalgia among patients with hepatitis B virus infection. Int. J. Clin. Exp. Med. 2013, 6, 804-808. [PubMed]

3. Pennisi, M.; Bertino, G.; Gagliano, C.; Malaguarnera, M.; Bella, R.; Borzì, A.M.; Madeddu, R.; Drago, F.; Malaguarnera, G. Resveratrol in Hepatitis C Patients Treated with Pegylated-Interferon- $\alpha-2 b$ and Ribavirin Reduces Sleep Disturbance. Nutrients 2017, 9, 897. [CrossRef] [PubMed]

4. Yoh, K.; Nishikawa, H.; Enomoto, H.; Iwata, Y.; Kishino, K.; Shimono, Y.; Hasegawa, K.; Nakano, C.; Takata, R.; Nishimura, T.; et al. Comparison of sleep disorders in chronic hepatitis $\mathrm{C}$ patients treated with interferon-based therapy and direct acting antivirals using actigraphy. Hepatol. Res. 2016, 46, 1358-1366. [CrossRef] [PubMed]

5. Mir, H.M.; Stepanova, M.; Afendy, H.; Cable, R.; Younossi, Z.M. Association of Sleep Disorders with Nonalcoholic Fatty Liver Disease (NAFLD): A Population-based Study. J. Clin. Exp. Hepatol. 2013, 3, 181-185. [CrossRef] [PubMed]

6. Erlangsen, A.; Runeson, B.; Bolton, J.M.; Wilcox, H.C.; Forman, J.L.; Krogh, J.; Shear, M.K.; Nordentoft, M.; Conwell, Y. Association Between Spousal Suicide and Mental, Physical, and Social Health Outcomes: A Longitudinal and Nationwide Register-Based Study. JAMA Psychiatry 2017, 74, 456-464. [CrossRef] [PubMed]

7. Chou, Y.T.; Cheng, H.J.; Wu, J.S.; Yang, Y.C.; Chou, C.Y.; Chang, C.J.; Lu, F.H. The association of sleep duration and sleep quality with non-alcoholic fatty liver disease in a Taiwanese population. Obes. Res. Clin. Pract. 2018, 12, 500-505. [CrossRef]

8. Labenz, C.; Baron, J.S.; Toenges, G.; Schattenberg, J.M.; Nagel, M.; Sprinzl, M.F.; Nguyen-Tat, M.; Zimmermann, T.; Huber, Y.; Marquardt, J.U.; et al. Prospective evaluation of the impact of covert hepatic encephalopathy on quality of life and sleep in cirrhotic patients. Aliment. Pharmacol. Ther. 2018, 48, 313-321. [CrossRef]

9. Bernsmeier, C.; Weisskopf, D.M.; Pflueger, M.O.; Mosimann, J.; Campana, B.; Terracciano, L.; Beglinger, C.; Heim, M.H.; Cajochen, C. Sleep Disruption and Daytime Sleepiness Correlating with Disease Severity and Insulin Resistance in Non-Alcoholic Fatty Liver Disease: A Comparison with Healthy Controls. PLoS ONE 2015, 10, e0143293. [CrossRef]

10. Iwasa, M.; Karino, Y.; Kawaguchi, T.; Nakanishi, H.; Miyaaki, H.; Shiraki, M.; Nakajima, T.; Sawada, Y.; Yoshiji, H.; Okita, K.; et al. Relationship of muscle cramps to quality of life and sleep disturbance in patients with chronic liver diseases: A nationwide study. Liver Int. 2018. [CrossRef]

11. Ghabril, M.; Jackson, M.; Gotur, R.; Weber, R.; Orman, E.; Vuppalanchi, R.; Chalasani, N. Most Individuals with Advanced Cirrhosis Have Sleep Disturbances, Which Are Associated with Poor Quality of Life. Clin. Gastroenterol. Hepatol. 2017, 15, 1271-1278. [CrossRef] [PubMed] 
12. Cordoba, J.; Cabrera, J.; Lataif, L.; Penev, P.; Zee, P.; Blei, A.T. High prevalence of sleep disturbance in cirrhosis. Hepatology 1998, 27, 339-345. [CrossRef] [PubMed]

13. Montagnese, S.; Middleton, B.; Mani, A.R.; Skene, D.J.; Morgan, M.Y. Sleep and circadian abnormalities in patients with cirrhosis: Features of delayed sleep phase syndrome? Metab. Brain Dis. 2009, 24, 427-439. [CrossRef] [PubMed]

14. Tordjman, S.; Chokron, S.; Delorme, R.; Charrier, A.; Bellissant, E.; Jaafari, N.; Fougerou, C. Melatonin: Pharmacology, Functions and Therapeutic Benefits. Curr. Neuropharmacol. 2017, 15, 434-443. [CrossRef] [PubMed]

15. Monaco, S.; Mariotto, S.; Ferrari, S.; Calabrese, M.; Zanusso, G.; Gajofatto, A.; Sansonno, D.; Dammacco, F. Hepatitis C virus-associated neurocognitive and neuropsychiatric disorders: Advances in 2015. World J. Gastroenterol. 2015, 21, 11974-11983. [CrossRef] [PubMed]

16. Montagnese, S.; Middleton, B.; Skene, D.J.; Morgan, M.Y. Night-time sleep disturbance does not correlate with neuropsychiatric impairment in patients with cirrhosis. Liver Int. 2009, 29, 1372-1382. [CrossRef] [PubMed]

17. Mostacci, B.; Ferlisi, M.; Baldi Antognini, A.; Sama, C.; Morelli, C.; Mondini, S.; Cirignotta, F. Sleep disturbance and daytime sleepiness in patients with cirrhosis: A case control study. Neurol. Sci. 2008, 29, 237-240. [CrossRef]

18. Mollayeva, T.; Thurairajah, P.; Burton, K.; Mollayeva, S.; Shapiro, C.M.; Colantonio, A. The Pittsburgh sleep quality index as a screening tool for sleep dysfunction in clinical and non-clinical samples: A systematic review and meta-analysis. Sleep Med. Rev. 2016, 25, 52-73. [CrossRef]

19. Buysse, D.J.; Reynolds, C.F., 3rd; Monk, T.H.; Berman, S.R.; Kupfer, D.J. The Pittsburgh Sleep Quality Index: A new instrument for psychiatric practice and research. Psychiatry Res. 1989, 28, 193-213. [CrossRef]

20. Doi, Y.; Minowa, M.; Uchiyama, M.; Okawa, M.; Kim, K.; Shibui, K.; Kamei, Y. Psychometric assessment of subjective sleep quality using the Japanese version of the Pittsburgh Sleep Quality Index (PSQI-J) in psychiatric disordered and control subjects. Psychiatry Res. 2000, 97, 165-172. [CrossRef]

21. Nishikawa, H.; Shiraki, M.; Hiramatsu, A.; Moriya, K.; Hino, K.; Nishiguchi, S. Japan Society of Hepatology guidelines for sarcopenia in liver disease (1st edition): Recommendation from the working group for creation of sarcopenia assessment criteria. Hepatol. Res. 2016, 46, 951-963. [CrossRef] [PubMed]

22. Montano-Loza, A.J. Clinical relevance of sarcopenia in patients with cirrhosis. World J. Gastroenterol. 2014, 20, 8061-8071. [CrossRef] [PubMed]

23. Cruz-Jentoft, A.J.; Landi, F.; Schneider, S.M.; Zúñiga, C.; Arai, H.; Boirie, Y.; Chen, L.K.; Fielding, R.A.; Martin, F.C.; Michel, J.P.; et al. Prevalence of and interventions for sarcopenia in ageing adults: A systematic review. Report of the International Sarcopenia Initiative (EWGSOP and IWGS). Age Ageing 2014, 43, 748-759. [CrossRef] [PubMed]

24. Sinclair, M.; Gow, P.J.; Grossmann, M.; Angus, P.W. Review article: Sarcopenia in cirrhosis-Aetiology, implications and potential therapeutic interventions. Aliment. Pharmacol. Ther. 2016, 43, 765-777. [CrossRef] [PubMed]

25. Chen, L.K.; Liu, L.K.; Woo, J.; Assantachai, P.; Auyeung, T.W.; Bahyah, K.S.; Chou, M.Y.; Chen, L.Y.; Hsu, P.S.; Krairit, O.; et al. Sarcopenia in Asia: Consensus Report of the Asian Working Group for Sarcopenia. J. Am. Med. Dir. Assoc. 2014, 15, 95-101. [CrossRef] [PubMed]

26. Nishikawa, H.; Enomoto, H.; Ishii, A.; Iwata, Y.; Miyamoto, Y.; Ishii, N.; Yuri, Y.; Hasegawa, K.; Nakano, C.; Nishimura, T.; et al. Elevated serum myostatin level is associated with worse survival in patients with liver cirrhosis. J. Cachexia Sarcopenia Muscle 2017, 8, 915-925. [CrossRef] [PubMed]

27. Norman, K.; Otten, L. Financial impact of sarcopenia or low muscle mass-A short review. Clin. Nutr. 2018. [CrossRef]

28. Shirai, H.; Kaido, T.; Hamaguchi, Y.; Kobayashi, A.; Okumura, S.; Yao, S.; Yagi, S.; Kamo, N.; Taura, K.; Okajima, H.; et al. Preoperative Low Muscle Mass and Low Muscle Quality Negatively Impact on Pulmonary Function in Patients Undergoing Hepatectomy for Hepatocellular Carcinoma. Liver Cancer 2018, 7, 76-89. [CrossRef]

29. De Bandt, J.P.; Jegatheesan, P.; Tennoune-El-Hafaia, N. Muscle Loss in Chronic Liver Diseases: The Example of Nonalcoholic Liver Disease. Nutrients 2018, 10, 1195. [CrossRef] 
30. Chang, K.V.; Chen, J.D.; Wu, W.T.; Huang, K.C.; Hsu, C.T.; Han, D.S. Association between Loss of Skeletal Muscle Mass and Mortality and Tumor Recurrence in Hepatocellular Carcinoma: A Systematic Review and Meta-Analysis. Liver Cancer 2018, 7, 90-103. [CrossRef]

31. Anderson, L.J.; Liu, H.; Garcia, J.M. Sex Differences in Muscle Wasting. Adv. Exp. Med. Biol. 2017, 1043, 153-197. [PubMed]

32. Yoo, S.Z.; No, M.H.; Heo, J.W.; Park, D.H.; Kang, J.H.; Kim, S.H.; Kwak, H.B. Role of exercise in age-related sarcopenia. J. Exerc. Rehabil. 2018, 14, 551-558. [CrossRef] [PubMed]

33. Goodpaster, B.H.; Park, S.W.; Harris, T.B.; Kritchevsky, S.B.; Nevitt, M.; Schwartz, A.V.; Simonsick, E.M.; Tylavsky, F.A.; Visser, M.; Newman, A.B. The loss of skeletal muscle strength, mass, and quality in older adults: The health, aging and body composition study. J. Gerontol. A Biol. Sci. Med. Sci. 2006, 61, 1059-1064. [CrossRef] [PubMed]

34. Trivedi, H.D.; Tapper, E.B. Interventions to improve physical function and prevent adverse events in cirrhosis. Gastroenterol. Rep. (Oxf.) 2018, 6, 13-20. [CrossRef] [PubMed]

35. Asahina, Y.; Tsuchiya, K.; Tamaki, N.; Hirayama, I.; Tanaka, T.; Sato, M.; Yasui, Y.; Hosokawa, T.; Ueda, K.; Kuzuya, T.; et al. Effect of aging on risk for hepatocellular carcinoma in chronic hepatitis $\mathrm{C}$ virus infection. Hepatology 2010, 52, 518-527. [CrossRef] [PubMed]

36. Abad, V.C.; Guilleminault, C. Insomnia in Elderly Patients: Recommendations for Pharmacological Management. Drugs Aging 2018, 35, 791-817. [CrossRef] [PubMed]

37. Kim, J.H.; Duffy, J.F. Circadian Rhythm Sleep-Wake Disorders in Older Adults. Sleep Med. Clin. 2018, 13, 39-50. [CrossRef]

38. Gulia, K.K.; Kumar, V.M. Sleep disorders in the elderly: A growing challenge. Psychogeriatrics 2018, 18, 155-165. [CrossRef]

39. Durazzo, M.; Belci, P.; Collo, A.; Prandi, V.; Pistone, E.; Martorana, M.; Gambino, R.; Bo, S. Gender specific medicine in liver diseases: A point of view. World J. Gastroenterol. 2014, 20, 2127-2135. [CrossRef]

40. Saner, N.J.; Bishop, D.J.; Bartlett, J.D. Is exercise a viable therapeutic intervention to mitigate mitochondrial dysfunction and insulin resistance induced by sleep loss? Sleep Med. Rev. 2018, 37, 60-68. [CrossRef]

41. Hussain, M.M.; Pan, X. Circadian regulators of intestinal lipid absorption. J. Lipid Res. 2015, 56, 761-770. [CrossRef] [PubMed]

42. Singh, J.; Sharma, B.C.; Puri, V.; Sachdeva, S.; Srivastava, S. Sleep disturbances in patients of liver cirrhosis with minimal hepatic encephalopathy before and after lactulose therapy. Metab Brain Dis. 2017, 32, $595-605$. [CrossRef] [PubMed]

(C) 2018 by the authors. Licensee MDPI, Basel, Switzerland. This article is an open access article distributed under the terms and conditions of the Creative Commons Attribution (CC BY) license (http://creativecommons.org/licenses/by/4.0/). 